\title{
An Efficient Learning Algorithm of Adaptive Beam Algorithms that is Establishing for Linear Array
}

\author{
D.Vimala, P.Nandhini, R. Elankavi
}

\begin{abstract}
The current cordless base that is cellular antenna system employs switched beam technology with fixed radiation sample. This science is affected with its inefficiency to trace the purchaser and ability that's restrained. The alternative strategies referred to as mobile sectoring and cellular splitting has acquired the essential dilemmas like more handoffs, further base position antennas and so on.The wise antenna technology which tracks an man or woman that's cellular by means of steering the important beam closer to an person as well because the same period of time setting up nulls within the recommendations of this interfering signal. The development of shrewd antennas entails the look of antenna array and adjusting the signal that's incoming altering the hundreds related to the amplitude aswell as period utilizing effective Digital $S$ ignal processing algorithms. This adaptive array guarantees to give most fulfilling solution for the aforementioned recounted issues and reinforce the ability associated with cordless process ability that's mobile. This challenge peculiarly pursuits Adaptive beam establishing algorithms such as for instance LMS and LMS/SMI will quickly be modified to appreciate one that is better amongst those two Algorithms predicated on price and precision for intelligent cell interaction base place antennas.
\end{abstract}

Keywords::Co-channel disturbance, Inter signal interference, variety, Least mean rectangular, recursive square that is mean $S$ ample matrix inversion, steady modulus algorithm.

\section{INTRODUCTION}

Within the last few years there is been a progress that's explosive the number of cordless users, primarily within the vicinity of mobile interplay. In future cordless programs being cellular become extra advanced and far more broad.

This development has brought about an demand that's colossal constrained to capability however moreover higher coverage and excessive best of resolution.A couple of company new technologies have now been explored and implemented on this respect to provide usage that's invaluable of limited assets. One good method to increase capacity is to apply the proposal of cellular technology, that entails dividing a coverage that is massive into little hexagonal cells. As a consequence, only one power that is high is converted with numerous low vigour transmitters. Each and every mobile is allocated a few regularity networks which can be unusual from those allotted to the cells which might be neighboring. Though the set that is same of may be reused through another cell provided that they're divided excellent adequate to not ever purpose disturbance. A colossal upsurge in capability is

Revised Manuscript Received on October 22, 2019.

* Correspondence Author

D.Vimala, Department of CSE, Bharath Institute of Higher Education and Research, Chennai, Tamilnadu, India.Email:vimalamuthu3@gmail.com

P.Nandhini, Department of CSE, Bharath Institute of Higher Education and Research, Chennai, Tamilnadu, India. Email:pnandhinisuresh@gmail.com

R. Elankavi, Department of CSE, Bharath Institute of Higher Education and Research, Chennai, Tamilnadu, India. Email:kavirajcse@gmail.com feasible for the reason that all these cells reuses the regularity variety...Nonetheless individual cordless communications is getting decidedly extra and rather more fashionable and it is carrying on with to cultivate at a fee that is exponential. Thus technologies being company new wanted inthe place of mobile communications to allow for potential that is future. Smart antennas or adaptive arrays these are dynamically in a position to adjust to the visitors that's altering. Intelligent antennas, quite often used for the period of the bottom position, radiate slim beams to provide users which can be quite a lot of. So long as the users are very good divided spatially the regularity that's targeted equal be reused, even when the United States ers had been within the same cell.

\section{SHREWD ANTENNAS}

Are most commonly tied to two foremost impairments in cell interaction methods, ability and gratification. They have got been multipath and co-channel disturbance. Multipath is a state of being which arises at any time when an indication that's sent expression from distinct obstacles inside the propagation atmosphere. This presents expand to indicators which will also be countless from various instructional materials. Due to the fact that the multipath signals comply with exclusive paths, they've more than a few levels when they are get to the receiver. The outcomes is degradation in alert first-rate when they're combined for the duration of the receiver for this reason of period mismatch. Co-channel disturbance could also be the disturbance between two signals that operate for the duration of the regularity that is precise same. The disturbance is typically because of a signal from an alternative cell occupying the designated equal regularity musical group in mobile interplay.

Sensible antenna is amongst the various promising technologies that may enable a greater potential in cordless sites by way of without problems multipath that's decreasing co-channel disturbance.

The complete system of mixing the indicators then concentrating rays in a path that's unique often referred to as electronic beam establishing. The first antenna that is smart had been created for used in defense force applications to suppress interfering or jamming alerts by way of the enemy. This technology had been lent to make use of to character cordless communications the place disturbance had been restricting the quantity of customers that a network might manage due to the fact disturbance suppression had been an element in this system. It is a assignment that is major practice intelligent antenna technological know-how to character cordless communications in view that that the site visitors is denser. 
They've been

- Switched beam

- Adaptive Arrays

The Switched beam method now could be easier set alongside the process that is completely adaptive. It offers an enhance that is gigantic approach capacity when comparing to traditional omnidirectional antenna systems or sector-situated programs.

The array that is adaptive would be the "smarter" of this two systems. This procedure tracks an person that is mobile by means of steerage the principal beam closer to an man or woman as good as the identical period of time establishing nulls into the instructions regarding the interfering sign. Like switched beam methods, moreover they integrate arrays. In most cases, the gotten signal from all of the spatially distributed antenna factors is accelerated by a weight. The hundreds are problematic in nature and modify the stage and amplitude. These alerts are mixed to provide the array output. These tricky loads are computed via an problematic algorithm that's adaptive which will also be pr e-programmed in to the $\mathrm{d}$ igital sign-processing unit that manages the sign radiated via the bottom position.

\section{ADAPTIVE BEAMFORMING}

Adaptive Beam forming is a procedure by which a variety of antennas is exploited to supply reception that's maximum a targeted direction $\mathrm{b}$ y calculating the signal arrival from a favored means (inside the existence of sound) even as signals of the same regularity off their recommendations are refused. That's attained by various the a whole lot of every o $f$ the sensors (antennas) determined within the array. It practically utilizes the speculation that, despite the fact that the alerts emanating from quite a lot of transmitters occupy the regularity that is detailed equal, they however arrive from various guidelines. This separation that is spatial exploited to separate your lives the distinct sign through the interfering alerts. In adaptive beam forming the masses which might be maximum iteratively computed making use of problematic algorithms in terms of various requisites.

Beam growing is usually completed $b$ y phasing the feed every single aspect of an array to make unique that alerts gotten or sent from all factors is more likely to be in stage in a method that's exact. The levels (the inter aspect stage) and most likely amplitudes are modified to optimize the gotten signal.

\section{LMS ALGORITHM}

Minimal suggest rectangular (LMS) algorithm is an algorithm that is adaptive which utilizes a gradient-based approach of steepest decent. LMS algorithm makes use of the fees of the gradient vector by means of the understanding which can be provided. LMS involves an method that's iterative makes successive corrections to your fat vector in direction of the bad associated with the gradient vector which finally outcome in the minimum recommend square error. In comparison with different algorithms LMS algorithm is

Particularly simple; it in most cases does no longer need correlation perform calculation nor does it require matrix inversions. Provide consideration to a Uniform Linear Array (ULA) with $\mathrm{N}$ isotropic factors, which types the predominant

subject of the $b$ that is adaptive setting up system as shown inside the determine beneath.

The creation of this antenna array is provided by using,

$$
\begin{aligned}
& w(n+1)=w(n)+\mu x(n)\left[d^{*}(n)-x^{h}(n) w(n)\right] \\
& =w(n)+\mu x(n) e^{*}(n) \\
& x(t)=s(t) a\left(\theta_{0}\right)+\sum_{i=1}^{u} u_{i}(t) a\left(\theta_{i}\right)+n(t)
\end{aligned}
$$

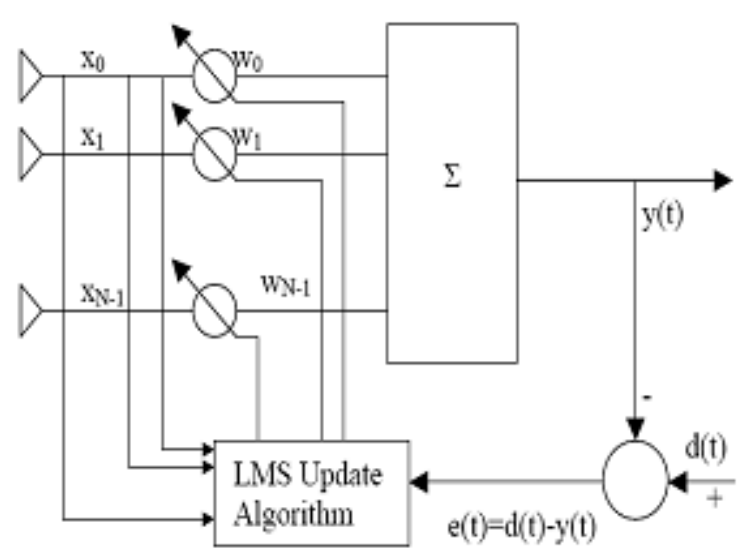

Figure 1: LMS adaptive procedure

$\mathrm{S}(\mathrm{t})$ denotes the required sign showing up at perspective $\theta 0$ and $t$ that's ui interfering signals accomplishing angle of incidences $\theta \mathrm{i}$ correspondingly. $\mathrm{A}(\theta 0)$ and $\mathrm{a}(\theta \mathrm{i})$ symbolize the $\mathrm{s}$ vectors that are teering the special signal and interfering alerts correspondingly. It is as a result needed to construct the targeted sign via the gotten $\mathrm{s}$ ignal amid the interfering signal and extra noise $n(t)$.

As shown over the outputs related to the exact sensors are linearly combined after being scaled making use of matching loads so that the antenna array pattern is optimized to possess highest reap that is conceivable the way in which involving the preferred sign and nulls in course of the interferers. The loads proper here may be computed LMS that's utilizing algorithm on minimal Squared Error (MSE) criterion. And so the filtering that is spatial entails estimation of sign through the gotten sign through minimizing the error between your guide signal, which intently suits or has some degree of correlation considering the fact that of the preferred sign estimate consequently the beam former production $y(t)$ (equal to $\left.\mathrm{w}^{*} \mathrm{x}(\mathrm{t})\right)$. This perhaps a Weiner that is ordinary filtering which is why the best solution is is also iteratively found out utilising the LMS algorithm.

Because of this fat enhance are allotted by the equation that is after where $\mu$ would be the step-dimension parameter and controls the convergence schools for the LMS algorithm.

On this algorith is established with an worth that's arbitrary0) for the additional weight vector at $n=z e r o$. The successive changes of thisfats vector basically contributes to the minimal price related to the imply mistake that's squared.

The LMS algorithm initiated with just a few worth that is arbitrary the additional weight vector typically appears to converge and remain steady forzero $<\mu \lambda$ that ismaximumcomputed by means of the SMI algorithm. 
The antenna beam has already been suggested to an approximate method of this desired signal prior to the LMS adaptation begins, with recognize to the preliminary fats estimate for the reason that SMI algorithm. Henceforth LMS part of this SMI/LMS algorithm takes time that's small converge.

\section{RESULTS AND DIALOG}

Then your algorithm converges tremendously slowly if $\mu$ is plumped for grow to be relatively small. A value that is significant of can result in a turbo convergence however is also less stable throughout the minimum worth.

\section{LMS WITH SMI INITIALIZATION ALGORITHM}

The simulation solutions are additionally offered to assess the efficiency associated with manufacturer new algorithm that is combined. The talks which might be prior LMS and SMI have definitely furnished us with an realization of those professionals and cons. Inside the mixed algorithm the in-patient good facets of both the algorithms are us ed.

LMS is an easy algorithm and it is suitable for consistent transmission methods as it's a algorithm that is consistently adaptive. Nevertheless, it is relatively not understood since of its convergence fee, which includes brought about participants to make use of different algorithms being complex once the Recursive least square (RLS) algorithm. SMI having said that has a particularly convergence that's rapid as talked about within the last chapter.

For this reason there was a necessity for an algorithm, which will also be an easy undertaking to put in force y et has a convergence that's fast and it is perhaps not computationally intensive. The algorithm $f$ eatured on this chapter is an effort in attaining this goal plus it will be named the SMI/LMS algorithm.

The SMI/LMS method talked about right here makes use of the deserves of each the LMS and also the SMI algorithm. The fashioned loads are decided utilising the SMI algorithm so that you could speed up convergence. The SMI algorithm achieves this by way of straight picking the inversion o $f$ the covariance matrix as illustrated by means of the equation that's after the masses determined correct listed here are just for 1 st few examples and even for $a b$ that's little of incoming information.

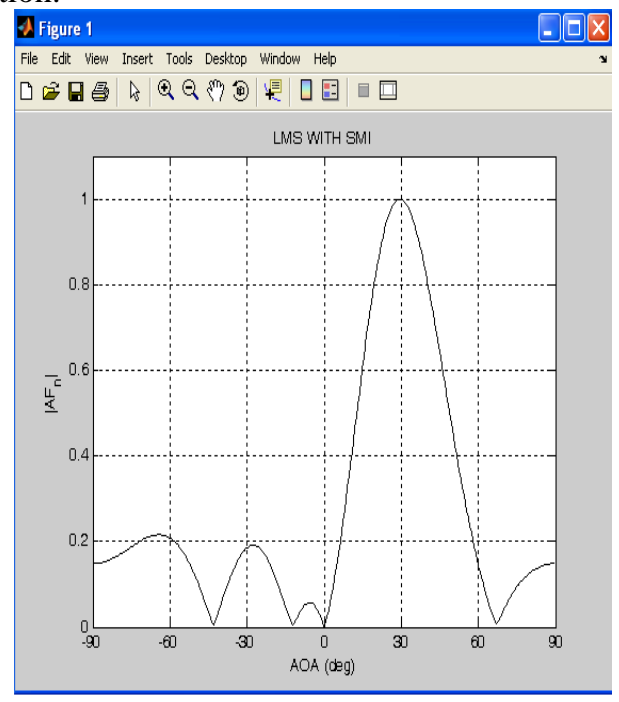

Figure.2.1:.Manner rectangular mistake
And so the fat that's initial equation may be re-written as Into the SMI/LMS algorithm w(0), the additional weight that's preliminary thewith preferred users AOA (in levels) 300 and Interferers AOA (in phases) 00. The algorithm converged at 55 th iteration it makes use of huge time wait via the simulated graph

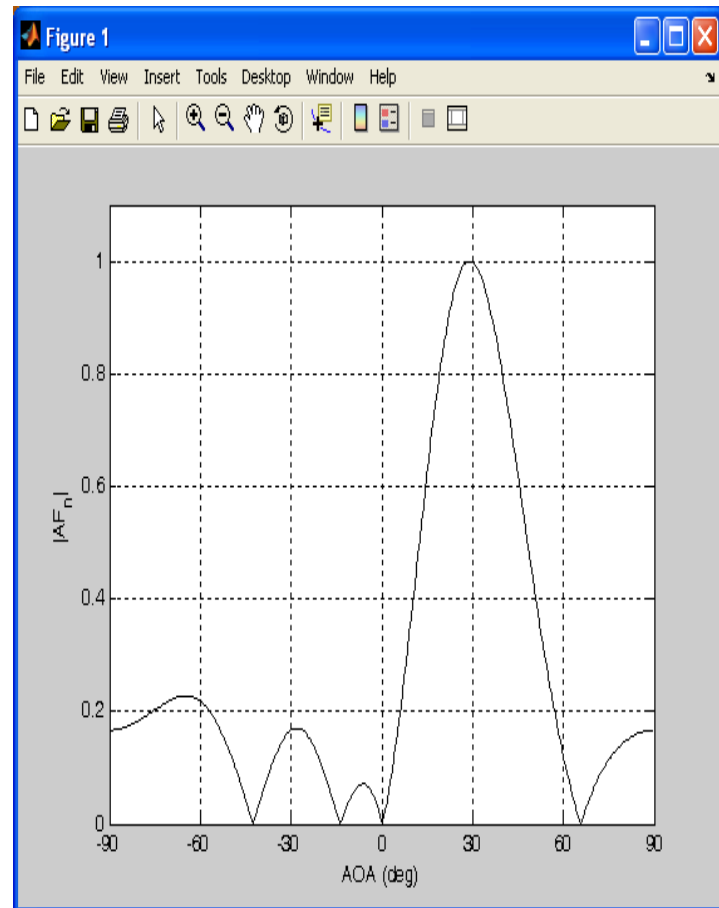

Figure 2.2: Weighted LMS arrayabove LMS alternate equation is akin to winreceived via the

SMI algorithm. And so the fats initialization for LMS isno precise arbitrary price $b$ an estimate uto $f$ the best price

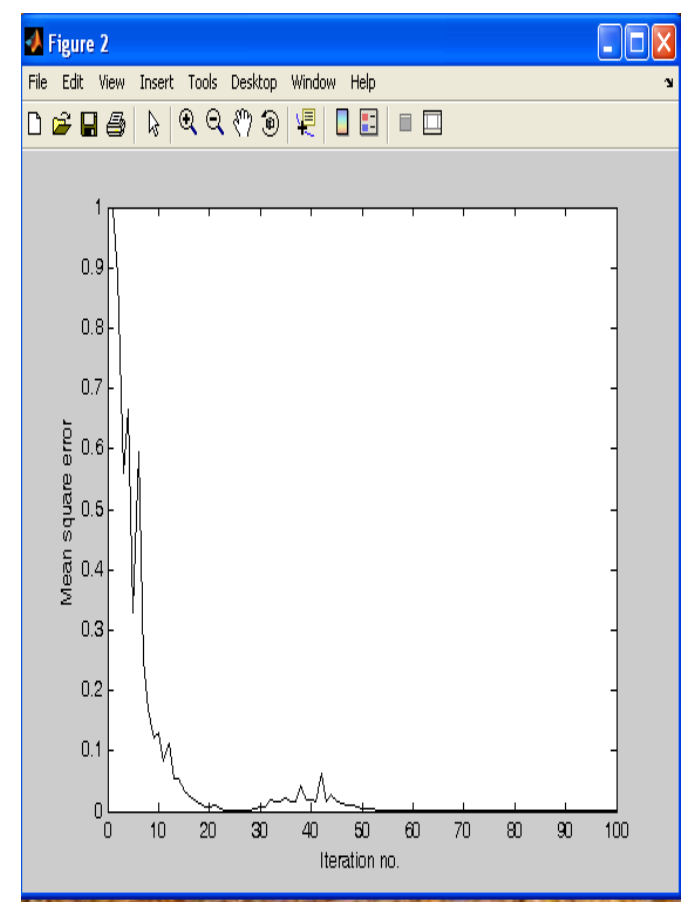

Figure.2.3:Weighted LMS/SMI array 


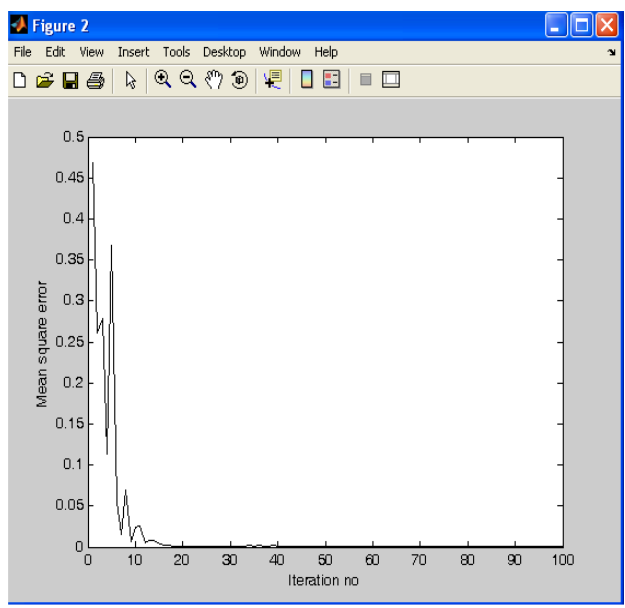

Figure.2.4:Approach rectangular mistake

\section{CONCLUSION}

we've simulated LMS and LMS/SMI algorithm for uniform linear array with 5 factors which might be isotropic making use of MATLAB. The figure published that the LMS/SMI has quick convergence rate over LMS. Intelligent antenna approach wanted quick convergence and screen the purchaser however each the algorithms require advisor sign so we must overview the convergence that is rapid processing algorithms like RLS, SMI, CMA and hybrid algorithms.

\section{REFERENCES}

1. Kumaravel A., Meetei O.N.,An application of non-uniform cellular automata for efficient cryptography,2013 IEEE Conference on Information and Communication Technologies, ICT 2013,V-,I-,PP-1200-1205,Y-2013

2. Kumarave A., Rangarajan K.,Routing alogrithm over semi-regular tessellations,2013 IEEE Conference on Information and Communication Technologies, ICT 2013,V-,I-,PP-1180-1184,Y-2013

3. Dutta P., Kumaravel A.,A novel approach to trust based identification of leaders in social networks, Indian Journal of Science and Technology,V-9,I-10,PP--,Y-2016

4. Kumaravel A., Dutta P.,Application of Pca for context selection for collaborative filtering,Middle - East Journal of Scientific Research,V-20,I-1,PP-88-93,Y-2014

5. Kumaravel A., Rangarajan K.,Constructing an automaton for exploring dynamic labyrinths, 2012 International Conference on Radar, Communication and Computing, ICRCC 2012,V-,I-,PP-161-165,Y-2012

6. Kumaravel A.,Comparison of two multi-classification approaches for detecting network attacks, World Applied Sciences Journal,V-27,I-11,PP-1461-1465,Y-2013

7. Tariq J., Kumaravel A.,Construction of cellular automata over hexagonal and triangular tessellations for path planning of multi-robots, 2016 IEEE International Conference on Computational Intelligence and Computing Research, ICCIC 2016,V-,I-,PP--,Y-2017

8. Sudha M., Kumaravel A.,Analysis and measurement of wave guides using poisson method,Indonesian Journal of Electrical Engineering and Computer Science,V-8,I-2,PP-546-548,Y-2017

9. Ayyappan G., Nalini C., Kumaravel A.,Various approaches of knowledge transfer in academic social network,International Journal of Engineering and Technology,V-,I-,PP-2791-2794,Y-2017

10. Kaliyamurthie, K.P., Sivaraman, K., Ramesh, S. Imposing patient data privacy in wireless medical sensor networks through homomorphic cryptosystems 2016, Journal of Chemical and Pharmaceutical Sciences .

11. Kaliyamurthie, K.P., Balasubramanian, P.C.An approach to multi secure to historical malformed documents using integer ripple transfiguration 2016 Journal of Chemical and Pharmaceutical Sciences 9

12. A.Sangeetha,C.Nalini,"Semantic Ranking based on keywords extractions in the web", International Journal of Engineering \&
Technology, 7 (2.6) (2018) 290-292

13. S.V.GayathiriDevi,C.Nalini,N.Kumar,"An efficient software verification using multi-layered software verification tool "International Journal of Engineering \& Technology, 7(2.21)2018 454-457

14. C.Nalini,ShwtambariKharabe,"A Comparative Study On Different Techniques Used For Finger - Vein Authentication", International Journal Of Pure And Applied Mathematics, Volume 116 No. 82017 , 327-333, Issn: 1314-3395

15. M.S. Vivekanandan and Dr. C. Rajabhushanam, "Enabling Privacy Protection and Content Assurance in Geo-Social Networks", International Journal of Innovative Research in Management, Engineering and Technology, Vol 3, Issue 4, pp. 49-55, April 2018.

16. Dr. C. Rajabhushanam, V. Karthik, and G. Vivek, "Elasticity in Cloud Computing", International Journal of Innovative Research in Management, Engineering and Technology, Vol 3, Issue 4, pp. 104-111, April 2018.

17. K. Rangaswamy and Dr. C. Rajabhushanamc, "CCN-Based Congestion Control Mechanism In Dynamic Networks", International Journal of Innovative Research in Management, Engineering and Technology, Vol 3, Issue 4, pp. 117-119, April 2018.

18. Kavitha, R., Nedunchelian, R., "Domain-specific Search engine optimization using healthcare ontology and a neural network backpropagation approach", 2017, Research Journal of Biotechnology, Special Issue 2:157-166

19. Kavitha, G., Kavitha, R., "An analysis to improve throughput of high-power hubs in mobile ad hoc network" , 2016, Journal of Chemical and Pharmaceutical Sciences, Vol-9, Issue-2: 361-363

20. Kavitha, G., Kavitha, R., "Dipping interference to supplement throughput in MANET", 2016, Journal of Chemical and Pharmaceutical Sciences, Vol-9, Issue-2: 357-360

21. Michael, G., Chandrasekar, A.,'Leader election based malicious detection and response system in MANET using mechanism design approach", Journal of Chemical and Pharmaceutical Sciences(JCPS) Volume 9 Issue 2, April - June 2016

\section{AUTHORS PROFILE}

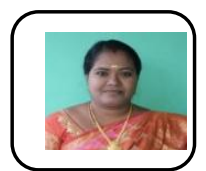

DVimala Assistant Professor, Department of Computer Science \& Engineering, Bharath Institute of Higher Education and Research, Chennai, India

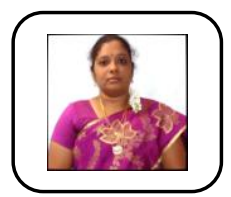

P.Nandhini Assistant Professor, Department of Computer Science \& Engineering, Bharath Institute of Higher Education and Research Chennai, India

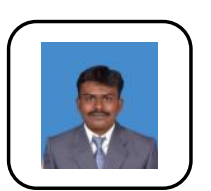

R.Elankavi Assistant Professor, Department of Computer Science \& Engineering, Bharath Institute of Higher Education and Research, Chennai, India 